\title{
DOSAGE DES LIPIDES \\ DANS LES PRODUITS ALIMENTAIRES ; \\ CONSIDÉRATIONS SUR LEUR VALEUR NUTRITIVE ( \\ PAR
}

\section{G. CLEMENT}

Chargé de Recherches au C.N.R.S.

\section{PLAN DU MÉMOIRE}

\section{PREMIËRE, PARTIE:}

Dosage des lipides.

A. - Estimarion du pourcentage de matières grasses dans les produits alimentaires.

B. - Isolement et dosage des constituants lipidiques, à partir d'un extrait lipidique total.

a) Isolement des phosphatides et dosage par leurs acides gras.

b) Séparation des acides gras libres.

c) Séparation quantitative des glycérides (et esters de cholestérol) de l'insaponifiable total.

C. - Analyse qualitative d'une huile ou d'une graisse.

\section{DEUXIËME PARTIE}

Valeur nutritive.

a) Digestibilité.

b) Rancidité.

c) Incidences biologiques ou physiologiques du traitement industriel des matières grasses alimentaires.

Le présent exposé comprendra deux parties distinctes :

- Une partie technique sur le dosage et l'analyse des lipides dans les produits alimentaires, y compris les matières grasses ;

- Une partie théorique comportant quelques considérations sur la valeur nutritive des lipides.

(1) Cours-conférence, donné le I6 avril 1956, à la Maison de la Chimie, sous les auspices du Centre de Perfectionnement Technique.

Annales de Zootechnie. - r956 


\section{PREMIËRE PARTIE}

\section{DOSAGE DES LIPIDES}

Le problème du dosage des lipides dans les produits alimentaires se présente de façon différente selon le but qu'on se propose. Il peut s'agir d'évaluer simplement la teneur en matières grasses d'un produit alimentaire donné ; c'est un problème de technologie alimentaire. On peut avoir à doser des constituants lipidiques particuliers, phosphatides par exemple. Il peut s'agir enfin de caractériser une huile ou une graisse et d'en étudier la composition. Nous envisagerons successivement ces divers points :

A. - Estimation du pourcentage de matières grasses dans les produits alimentaires.

B. -- Dosages des constituants lipidiques à partir d'un extrait lipidique total,

C. - Analyse qualitative d'une huile ou d'une graisse.

\section{A. - ESTIMATION DU POURCENTAGE DE MATIERE GRASSES DANS LES PRODUITS ALIMENTAIRES}

Nous nous contenterons pour l'instant de définir "l'extrait lipidique total " comme étant l'ensemble des substances reprises par un solvant organique anhydre après extraction à l'alcool bouillant. Cet extrait contient en plus des " lipides » proprement dits, des substances qui les accompagnent comme les stérols, les hydrocarbures et certaines vitamines liposolubles. Le groupe des lipides est en effet caractérisé par son insolubilité dans l'eau et sa solubilité dans les solvants organiques : éther, chloroforme, benzène, etc...

Fn principe, lorsqu'on a à faire à des tissus animaux qui ont une teneur en eau assez importante (50 à 75 p. 10o) une teneur en glucides faible et dont les lipides sont plus ou moins liés aux protéines sous forme de cénapses, la méthode la plus rigoureuse est la suivante :

a) précipitation des complexes lipoprotéiques à l'alcool à $95^{\circ}$ d'un échantillon représentatif du tissu à analyser, préalablement coupé aux ciseaux ou broyé au mortier ;

b) extraction au Soxhlet ou Kumagawa par 1'alcool à $95^{\circ}$, à ébullition pendant 6 ou 7 heures ;

c) réunion des alcools de précipitation et d'extraction, évaporation et séchage sous vide à l'alcool absolu ;

d) reprise de l'extrait lipidique par un solvant organique anhydre (benzène, chloroforme, éther sulfurique) au-dessus d'un filtre amiante- 
sable. Après évaporation du solvant, le résidu est pesé à poids constant et constitue l'extrait lipidique total.

Cette méthode idéale pour tous les tissus animaux peut être appliquée évidemment sans difficultés en matière d'alimentation aux viandes fraîches ou de conserves, ainsi qu'à de nombreuses préparations à base de viande. Cependant, dans certains cas, le dosage des lipides dans les produits alimentaires présente des difficultés en raison de l'hétérogénéité du produit, de son état physique, de son degré d'hydradation. Mais, en fait, la précision qu'on attend des dosages n'est pas très grande dans la plupart des cas et les résultats obtenus par les diverses méthodes sont en gros équivalents. Nous donnerons seulement un rapide aperçu des particularités de dosages propres à certaines catégories de produits alimentaires en envisageant successivement : l'échantillonnage, la dessiccation, l'extraction des lipides.

\section{a) L'échantillonnage.}

On ne saurait trop insister sur ce premier temps qui est évidemment très important; il faut que l'échantillon soit représentatif du produit à étudier. Il s'agit de cas d'espèces et on ne peut donner de règle générale; les dimensions de l'échantillon à prélever dépendent du degré d'hétérogénéité du produit. I'échantillonnage pour un saucisson, par exemple, va dépendre de la dimension et du nombre de cubes de lard qui y sont introduits; pour une boîte de pâté il faudra tenir compte de la barde de graisse qui l'entoure. L'échantillonnage correct d'un fromage devra comporter plusieurs tranches prélevées à des endroits différents selon ses rayons et comprenant la croûte, les tranches sont ensuite morcelées finement, mélangées et écrasées. Ce deuxième temps "d'homogénéisation » du produit est également très important. Très souvent, en charcuterie notamment, il est utile de broyer la substance (pâté, saucisse, etc...) en présence de sable fin afin de faciliter 1'extraction ultérieure. En biscuiterie, SARAzIN et coll. (34) insistent dans un article récent, sur l'importance du degré de broyage, de la granulation, pour les dosages de graisses dans les biscottes.

\section{b) La dessiceation.}

Le plus souvent 1'extraction des lipides est faite sur un produit desséché. I.es méthodes A. O. A. C. publiées en I940-I945 prévoient en effet avant l'extraction une dessiccation des prises d'essai par un séjour à l'étuve

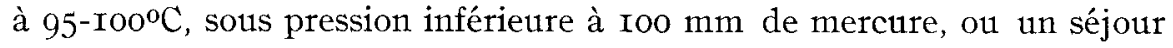
dans un dessiccateur sous vide en présence d'acide sulfurique. On utilise aussi très souvent $\mathrm{SO}_{4} \mathrm{Na}_{2}$ anhydre pour dessécher les produits. 


\section{c) L'extraction.}

L'alcool à $95^{\circ}$ qui est préconisé dans le cas d'un produit frais, non desséché, ne convient pas pour une substance séchée ; ou extrait alors au Kumagawa ou au Soxhlet pendant 6 heures avec un solvant des graisses : benzène, chloroforme ou éther sulfurique. Après filtration et évaporation du solvant, on pèse à poids constant le résidu sec qui représente les lipides totaux.

Hydrolyse acide. - Dans différents cas, lorsque les lipides ne contiennent pas de phosphatides - en fromagerie par exemple - ou lorsque le pourcentage de graisse est faible (pain ou biscottes), au lieu d'extraire les lipides totaux, on procède à une hydrolyse chlorhydrique ou sulfurique (comme dans la méthode de Schmm-Bondzynski Ratslaff) qui libère ainsi les acideś gras ; on extrait ensuite à 1'éther sulfurique-éther de pétrole dans une ampoule à décantation. On filtre et après évaporation on sèche l'extrait lipidique total et on pèse. Il serait plus correct, à notre avis, de reprendre, après séchage à l'alcool absolu, le résidu lipidique par un solvant anhydre. Dans le calcul du poids des lipides obtenus, on devrait aussi tenir compte de la part revenant au glycérol, car après hydrolyse on ne pèse, en fait, qu'un mélange d'acides gras.

Méthode industrielle rapide. - Avec des appareils comme l'humidimètre "Gallia " par exemple, on peut faire des déterminations rapides (produits de charcuterie par exemple) des teneurs en eau et en matières grasses à quelques pour cent près. Les deux opérations demandent en tout une heure et demie avec un minimum de manipulations. Le principe est très simple : il s'agit en fait d'une balance dont le plateau est placé dans une enceinte qu'on peut chauffer à lampe infra-rouge ou même à simple filament de carbone. On mesure la perte de poids par évaporation en lisant simplement le déplacement d'un spot lumineux sur une échelle graduée. Dans un premier temps on s'en sert comme humidimètre : on obtient donc un extrait sec; on rince ensuite sur un entonnoir cet extrait sec plusieurs fois à l'éther sulfurique et on le replace dans l'appareil, on fait alors une deuxième lecture sur l'extrait sec ainsi délipidé après évaporation complète de l'éther pour l'obtention du pourcentage de matières grasses.

Cas du lait. - Le problème du dosage des lipides du lait revêt un caractère à la fois économique et pratique. Economique, puisque le prix d'achat du lait est en partie fonction de sa teneur en matières grasses, d'où la nécessité de le doser avec grande précision; pratique, car il faut une méthode rapide et propice aux dosages en série.

On trouvera une mise au point toute récente sur la question dans 
l'article de PIEN (3I), aussi ne donnerons-nous ici que le principe des méthodes les plus utilisées.

Il existe deux méthodes pondérales de dosage : la méthode internationale de Röse GotTLIEB considérée comme méthode étalon et la méthode officielle française, et une méthode volumétrique couramment employée en industrie laitière : la méthode de GERBER.

Dans la méthode de Röse GotTLIEB, les lipides sont libérés par 1'amnoniaque et l'alcool, puis on fait des extractions successives à l'éther sulfurique et ensuite à l'éther de pétrole. On réunit les liqueurs d'extractions, on évapore, on sèche et on pèse. Cette méthode est précise à $\pm 0, \mathrm{I} g$ de matières grasses par litre mais ne convient que pour le lait liquide normal en bon état de conservation. Si le lait contient des acides gras libres, ceux-ci formant des savons avec l'ammoniaque, restent dans la phase aqueuse et échappent aux dosages.

Dans la méthode officielle française, la libération des graisses est obtenue par une solution aqueuse diluée d'acide acétique à 2 p. Ioo. On extrait ensuite à 1'éther.

I a méthode de GERBER consiste, après dissolution des protéines par de l'acide sulfurique et addition d'alcool amylique pour faciliter le rassemblement des matières grasses et centrifugation du mélange, à évaluer le volume de graisse directement dans le tube de centrifuge. Cette méthode qui comporte des causes d'erreur par défaut (perte de matières grasses dans la phase acide) ou par excès (impuretés amyliques et sulfuriques) ainsi que des erreurs dues à la graduation des butyromètres, semble cependant donner satisfaction dans la pratique si l'on ne veut pas une précision supérieure à $\pm 0,5 \mathrm{~g}$ de M. G. par litre.

\section{B. - ISOLEMENT ET DOSAGE DES CONSTITUANTS LIPIDIQUES A PARTIR D'UN EXTRAIT LIPIDIQUE TOTAL}

Dosage et isolement n'ont de sens évidemment que s'ils sont pratiqués sur un extrait lipidique total obtenu dans de bonnes conditions : extraction à l'alcool chaud au Kumagawa ou au Soxhlet. La dessiccation à l'étuve doit être écartée, car elle peut entraîner une hydrolyse des constituants lipidiques ou une altération des acides gras. Les techniques que nous allons décrire sont classiques et permettent un dosage rigoureux des constituants lipidiques ; une longue pratique nous a permis d'en éprouver la validité. Ces techniques comportant l'isolement préalable des divers constituants permettent après leur hydrolyse l'analyse ultérieure des $\mathrm{A}$. G. de chaque fraction (indice d'iode, indice de saponification, spectrophotométrie,etc).

L'extrait lipidique total peut être constitué par des phosphatides, des glycérides, des A. G. libres, des esters de cholestérol et des substances insaponifiables (stérols, hydrocarbures, vitamines). 


\section{a) Isolement des phosphatides et dosage par leurs acides gras.}

L'extrait lipidique total est dissous dans un faible volume de benżène, de chloroforme ou d'éther anhydre et les phosphatides sont précipités par addition d'acétone anhydre ( 9 vol. pour I vol. de benzène, chloroforme ou éther) en présence de quelques gouttes d'une solution saturée de $\mathrm{MgCl}_{2}$ dans l'alcool absolu. Après un séjour de 6 heures à la glacière, la totalité des phosphatides a précipité ; on centrifuge à $4200 \mathrm{t} / \mathrm{m}$ pendant 20 minutes. Pour purifier encore les phosphatides, il est bon d'effectuer une deuxième précipitation.

Le précipité est ensuite saponifié par addition d'une solution $2 \mathrm{~N}$ de $\mathrm{KOH}$ dans l'alcool à $95^{\circ}\left(25 \mathrm{~cm}^{3} \mathrm{p}\right.$. I g de précipité $)$ pendant 2 heures au bainmarie bouillant. Après addition d'eau pour abaisser la teneur en alcool du milieu, on acidifie avec $\mathrm{HCl}$ au $\mathrm{I} / 3$ et on extrait les A. G. par 3 extractions à 1'éther sulfurique. Les phases éthérées sont réunies, séchées à 1'alcool absolu et les acides gras sont repris par de l'éther de pétrole anhydre (pt E $\left.30^{-} 5^{\circ}\right)$ au-dessus d'un filtre amiante-sable. Après évaporation on les pèse à poids constant.

Remarque. - On peut évaluer les phosphatides par leur phosphore soit à partir de l'extrait lipidique total, soit à partir du précipité de phosphatides. Après minéralisation, on dose le $\mathrm{P}$ par une méthode colorimétrique (MAcheboEUf-Delsal (26)) par exemple.

\section{b) Séparation des acides gras libres.}

Après avoir précipité les phosphatides, le liquide acétonique qui surnage contenant des acides gras libres, glycérides, esters de cholestérol et insaponifiable, est évaporé à sec. On reprend les lipides par de l'éther de pétrole et on fait 2 extractions à la potasse $\mathrm{N} /$ ro dans 1 'alcool à $50^{\circ}$, puis 3 extractions de la phase hydroalcoolique par de l'éther de pétrole (pt $\mathrm{E}=30^{\circ}-70^{\circ}$ ). Les acides gras libres forment des savons et restent dans la phase éther de pétrole (LEMELAND (23)). La phase hydroalcoolique est mise à évaporer (réduction de $\mathrm{I} / 3 \mathrm{du}$ volume) et après acidification avec $\mathrm{HCl}$ au $\mathrm{r} / 3$, on extrait les $\mathrm{A}$. G. comme précédemment.

\section{c) Séparation quantitative des glycérides (et esters de cholestérol) de l'insaponifiable total (technique de LEMELAND (23))}

I a phase éther de pétrole est évaporée. On saponifie à reflux avec une solution de $\mathrm{KOH} 2 \mathrm{~N}$ dans l'alcool à $95^{\circ}\left(25 \mathrm{~cm}^{3}\right.$ pour I $g$ de lipides $)$, pendant 2 heures au bain-marie bouillant. On ajoute ensuite $\mathrm{HCl}$ normal, eau et alcool à $95^{\circ}$, pour obtenir un milieu $\mathrm{N} / \mathrm{I}$ o dans $1^{\prime}$ alcool à $50^{\circ}$, seul 
milieu permettant de séparer les A. G. de l'insaponifiable. Si l'on utilise $25 \mathrm{~cm}$ de $\mathrm{KOH}$ au départ, on ajoute $40 \mathrm{~cm}^{3} \mathrm{de} \mathrm{HCl} \mathrm{N}, 26 \mathrm{~cm}^{3}, 2$ d'alcool à $95^{\circ}$ et $8 \mathrm{~cm}^{3}, 8$ d'eau.

On fait bouillir à reflux pendant 20 minutes encore, puis on extrait l'insaponifiable par l'éther de pétrole $30^{\circ}-70^{\circ}$. Les A. G. appartenant aux glycérides (et aux esters de cholestérol s'il s'agit de tissus animaux) passent dans la phase hydroalcoolique où ils forment des savons, on extrait comme précédemment, l'insaponifiable reste dans l'éther de pétrole, après évaporation et séchage, on reprend par de l'éther de pétrole anhydre; on le sèche et l'on pèse à poids constant.

\section{d) Dosage du cholestérol.}

Méthode gravimétrique. - C'est la méthode classique de WindausCAMinade ( 7$)$, on dose le cholestérol sous forme de complexe avec la digitonine; on évalue le cholestérol libre dans l'extrait lipidique total et le cholestérol total dans l'insaponifiable total obtenu après saponification.

Méthodes colorimétriques. - Elles sont utilisées pour de petites quantités de cholestérol (le cholestérol estérifié ou total dissous dans le chloroforme donne en présence d'acide sulfurique la réaction colorée de LIFBERMann-Burchard). Citons parmi ces méthodes celle de MacheboeurDELSAL (25) et celle de SPERRY (36); cette dernière est particulièrement intéressante car elle ne comporte pas les causes d'erreurs par excès des autres méthodes colorimétriques - le dosage colorimétrique porte ici sur le complexe digitonine-cholestérol, préalablement isolé par centrifugation.

Les phytostérols peuvent être dosés par leurs digitonides et séparés du cholestérol à partir de ces digitonides par formation d'acétates qui ont des points de fusion différents.

\section{MÉTHODES CHROMATOGRAPHIQUES}

Ces méthodes ont été préconisées récemment pour isoler les constituants lipidiques. Nous ne ferons ici que les citer:

a) séparation des divers constituants lipidiques sur silice (BORGSTRÖM (4)).

b) isolement des A. G. libres sur résine échangeuse d'ions (amberlite), DesNueliess (35), Borgström (4).

c) isolement des esters de cholestérol (Clément et coll. (8)).

d) chromatographie des phosphatides (LEA et RHODES (2I)).

e) séparation des glycérides partiels (DESNUELLLES(35), BORGSTRöM(5)).

Signalons enfin l'emploi de techniques chromatographiques particulières dans l'analyse des huiles et même pour leur purification à l'échelle 
industrielle. On a des méthodes pour séparer et doser le carotène (COOLEY KoEHN (Io), MÉLIER (29)), et pour séparer les graisses neutres des acides gras libres (DESNUELLES et NAUDET (II)).

Nous citerons dans le chapitre suivant celles qui concernent le fractionnement des acides gras.

Nous ferons une remarque en ce qui concerne l'emploi des méthodes chromatographiques en général, appliquées à l'isolement des constituants lipidiques : lorsqu'on met au point une méthode, on utilise généralement des mélanges synthétiques et semi synthétiques. Or, s'il est parfois aisé de séparer les constituants qu'on a ainsi mélangés, dès qu'on veut appliquer la technique à des extraits lipidiques de composition connue mais provenant de tissus, il n'en va pas toujours de même. Il faut donc être très prudent et ne pas généraliser trop vite en matière de chromatographie des lipides.

Enfin un danger qu'il faut aussi signaler, c'est le fait que si l'élution n'est pas quantitative, les fractions qui passent et celles qui sont retenues n'ont pas la même composition. Il en est ainsi, nous l'avons constaté, pour la séparation des acides gras libres sur amberlite par comparaison avec leur séparation par voie chimique (9).

\section{C. - ANALYSE QUALITATIVE D'UNE HUILE OU D'UNE GRAISSE}

Bien qu'il n'existe pas de critère scientifique permettant de distinguer une huile d'une graisse, on utilise couramment le premier terme pour désigner une matière grasse liquide à la température ordinaire et le second pour désigner une matière grasse solide. Les huiles et graisses alimentaires sont constituées principalement de triglycérides, c. a .d. de glycérol combiné de 3 molécules d'acides gras ; ces 3 molécules sont rarement d'une même sorte ce qui fait que les triglycérides sont généralement des glycérides mixtes (I4). Les matières grasses naturelles contiennent aussi parfois de petites quantités de monoglycérides ainsi que des acides gras libres, des phosphatides et des substances insaponifiables - (hydrocarbures, stérols et vitamines liposolubles).

Rappelons que les acides gras entrant dans la constitution des lipides naturels sont presque exclusivement des acides à chaîne droite et à nombre pair d'atomes de carbone allant de $\mathrm{C}_{4}$ à $\mathrm{C}_{24}$. Certains comme les acides palmitique, stéarique et oléique sont particulièrement fréquents. On distingue parfois différents groupes d'huiles ou de graisses qui semblent avoir une parenté de constitution ; c'est ainsi que l'on parle de groupe butyrique, de groupe laurique, de beurres végétaux, de graisses animales. Il faut cependant remarquer que le fait de connaître la composition exacte en acides gras d'une huile ou d'une graisse, en admettant que la chose soit toujours possible ne suffit pas à la définir, d'une part à cause de la présence 
des constituants non glycéridiques, d'autre part à cause du polymorphisme même des glycérides naturels (MALKIN (I6)). On peut donc dire que 1'analyste n'aura jamais trop de critères physiques ou chimiques à sa disposition pour caractériser une matière grasse. Comme il s'agit bien souvent de tests plus ou moins empiriques, on n'insistera jamais trop non plus sur la nécessité de les appliquer avec minutie et dans des conditions rigoureusement définies. C'est l'interprétation de l'ensemble des résultats obtenus par ces tests qui permettra à un technicien expérimenté de faire le "diagnostic " d'une huile ou d'une graisse. Il va de soi qu'il serait parfaitement absurde de se livrer à ces recherches qui sont souvent longues et délicates sur un échantillon qui ne serait pas parfaitement représentatif.

Ceci dit, quels sont les tests analytiques utilisés? I1 en existe un nombre tellement considérable que nous ne ferons qu'énumérer les principaux. I1 existe de nombreuses méthodes physiques pour déterminer le point de fusion, le point de solidification et le titre, la consistance, la viscosité, la densité, l'indice de réfraction, etc... On en trouvera l'exposé détaillé dans des revues spécialisées (14) (4I) (38).

Nous dirons seulement un mot des méthodes chimiques les plus courantes, car elles sont d'un usage très général en matière de Biochimie des lipides:

a) l'indice de saponification (quantité de potasse en $\mathrm{mg}$ nécessaire pour saponifier I $g$ de corps gras) renseigne sur le poids moléculaire moyen des acides gras. L'équivalent de saponification est le nombre de grammes de substances saponifiées par 56 , I mg (un équivalent de $\mathrm{KOH}$ ). On utilise de la potasse alcoolique $2 \mathrm{~N}$ et on dose en présence de phénolphtaléine à 0, 2 p. Ioo dans l'alcool ; 1'acide qui sert à titrer la potasse doit être étalonné. I'ébullition ne doit pas être prolongée trop longtemps sinon on risque la formation de réactions secondaires, entraînant une diminution de l'indice (PAquOT (30)).

b) l'indice d'iode (nombre de grammes d'iode fixés par $\mathrm{g}$ de graisse) renseigne sur le degré de désaturation des A. G. c. a. d. sur le nombre global des liaisons éthyléniques. I a méthode de WiJs est excellente à condition de préparer convenablement les réactifs et d'étalonner la solution d'hyposulfite avec le bichromate.

c) l'acidité libre : on la mesure le plus souvent par simple titration à la potasse alcoolique et on l'exprime en pourcentage d'A. G. libres, calculé sur la base de l'acide gras libre prédominant : oléique, laurique, etc., selon le cas. Par titration, le test n'est pas très sensible.

d) l'indice d'hydroxyle rend compte des fonctions alcool non estérifiées existant dans un corps gras. C'est le nombre de mg de potasse nécessaires pour neutraliser l'acide acétique qui se combine par acétylation à I g de 
M. G. La mesure de cet indice rend compte des monoglycérides et aussi des acides gras hydroxylés qui peuvent apparaître dans un corps gras altéré.

e) Les acides gras volatils, de bas poids moléculaires, peuvent être estimés par les indices de REICHERT (A. G. votatils solubles) de POLENSKE (A. G. votatils insolubles) et de Kirschner (A. G. butyrique). Tous ces indices s'expriment en $\mathrm{mm}^{3}$ de solution $\mathrm{O}, \mathrm{I} \mathrm{N}$ de soude nécessaires pour neutraliser les $\mathrm{A}$. G. distillés à partir de $5 \mathrm{~g}$ de graisse dans des conditions bien définies.

f) Le degré d'oxydation d'une graisse est évalué classiquement par la méthode de Lea (oxydation de l'iodure de potassium à froid ou à chaud par les peroxydes). I'indice de LEA est le nombre de microgrammes d'oxygène fixé sous forme de peroxydes par I $g$ de corps gras. Cette méthode peut être rendue plus sensible par addition de thiofluorescéine (DuBouLoz (I3)).

\section{$* *$}

Enfin nous voudrions faire une place à part à une méthode récente appelée certainement à un grand développement : la spectrophotométrie ultra-violette. Cette méthode est particulièrement intéressante pour le nutritionniste parce qu'elle met à sa disposition un moyen élégant de dosage (dans certaines conditions) des acides gras polyéthyléniques, donc des A. G. indispensables. Le principe en est le suivant : en l'absence de doubles liaisons conjuguées, les A. G. ne possèdent pas de bandes d'absorption dans $1^{\prime} U$. V. Après isomérisation alcaline par chauffage à $\mathrm{I} 80^{\circ}$, en présence d'une solution glycolique de potasse les acides linoléiques, linoléniques et arachidoniques isomérisés, présentent alors des bandes d'absorption avec des pics caractéristiques $234 \mathrm{~A}^{\circ}$ pour le linoléique, $268 \mathrm{~A}^{\circ}$ pour le linolénique et $3 \mathrm{I} 6 \mathrm{~A}^{\circ}$ pour l'arachidonique.

On n'a pas encore pu déterminer les bandes d'absorption caractéristiques pour les pentaènes et les héxaènes puisqu'il n'a pas été possible de les isoler à l'état pur. D'ailleurs la présence de ces hauts polyènes dans un mélange d'acides gras désaturés rend les calculs pratiquement impossibles ; cette méthode n'est pas encore mise au point définitivement et ses conditions d'application ne sont pas encore pafaitement déterminées. Des facteurs de correction sont nécessaires notamment pour le fond spectral (May (28)). Quoiqu'il en soit elle rend d'ores et déjà de grands services et donne des résultats certainement valables dans un grand nombre de cas.

Jusqu'à ces dernières années, on ne possédait pour établir la composition en acides gras d'une huile ou d'une graisse que les méthodes mises au point par HiLDitch et ses élèves. Ces méthodes longues et laborieuses 
consistent à faire des cristallisations fractionnées des acides gras dans divers solvants à basse température, acétone à $-60^{\circ},-40^{\circ}$, éther à $-35^{\circ}$. Après avoir ainsi recueilli 4,5 ou 6 fractions d'acides gras selon les cas, on les transforme en esters méthyliques qui sont ensuite soumis à des distillations fractionnées sous vide. Les sous-fractions d'esters méthyliques obtenues sont saponifiées ; on libère les acides gras et on en détermine l'indice d'iode et l'indice de saponification de façon très rigoureuse. On pratique sur les fractions les plus désaturées des indices de brome ou mieux des mesures spectrophotométriques U. V. pour connaître la composition en acides linoléiques et linoléniques.

On doit enfin, après toutes ces manipulations effectuer des calculs longs et compliqués en se basant sur des tables de références établies à partir d'acides gras purs dont on a déterminé les constantes.

Ces méthodes qui sont utilisées dans un grand nombre de laboratoires du monde entier, tendent peu à peu à perdre leur place devant des techniques plus modernes rapides et élégantes, techniques chromatographiques ou techniques de distribution à contre-courant.

Nous ne ferons que citer quelques-unes de ces techniques, la plupart étant encore à l'étude.

Rappelons que c'est la chromatographie de partage gaz-liquide inventée par JAMES et MARTIN (I8) qui a été décrite d'abord pour la séparation des acides gras volatils, puis celle de Howard et MARTIN ( $I 7$ ) pour la séparation des acides gras supérieurs.

Une méthode qui semble aussi donner des résultats est celle développée par Boldingh ( 3 bis) aux Pays-Bas. Il s'agit encore de chromatographie de partage dans laquelle le support est constitué par du caoutchouc finement pulvérisé, la phase stationnaire est le benzène ou le chloroforme. On passe les acides gras dans du benzène et on élue avec des mélanges à base d'eau saturée de benzène, d'alcool méthylique et d'acétone. Les auteurs obtiennent ainsi une bonne séparation des acides gras saturés de C. 6 à C. I 8 ainsi que des A. C. gras en C. I 8 mais celle des acides gras saturés et désaturés de poids moléculaires différents est difficile.

La chromatographie sur papier a même été essayée par plusieurs auteurs.

Enfin la distribution à contre-courant a été développée surtout par Craig (I). Ce genre de technique est particulièrement souhaitable pour la séparation des lipides car il constitue un moyen doux de séparation par comparaison avec les distillations et même avec la chromatographie. CraIG et ses collaborateurs ( $\mathrm{I}$ ) ont obtenu ainsi de très bonnes séparations des acides gras saturés supérieurs ainsi qu'une séparation très correcte des acides gras en $\mathrm{C}$. I8. Les auteurs disent avoir trouvé de cette manière, à partir d'une graisse mésentérique de porc, des résultats concordants avec les analyses spectrophotométriques pour les acides gras en $\mathrm{C}$. I8, et avec 
les tableaux de composition établis anciennement par HiLditch pour les acides gras saturés. Un des avantages encore et non des moindres, de ces techniques de distribution à contre-courant est qu'elles peuvent être effectuées en continu dans des appareils complètement automatiques.

Il ne fait pas de doute que ces méthodes nouvelles, quand elles seront définitivement mises au point, rendront de très grands services à la biochimie des lipides. On peut donc espérer connaitre dans un très proche avenir d'une façon rapide et élégante, la composition exacte en acides gras d'un constituant lipidique quelconque. Mais la fraction d'acide gras la plus difficile à obtenir restera sûrement celle des acides gras supérieurs à grand nombre de doubles liaisons, les hauts polyènes. Leur séparation serait extrêmement importante, car elle permettrait d'étendre le domaine de la spectrophotométrie $U$. V. Elle nous renseignerait mieux aussi sur la composition des lipides de certains tissus, le tissu nerveux en particulier. Il faut ajouter encore le fait que dans les molécules de constituants lipidiques naturels, les acides gras doivent être disposés dans un certain ordre. Il doit y avoir certains types de structure de sorte que la seule composition en acides gras ne suffit pas à les définir. Et ceci à notre avis est certainement important du point de vue biologique : nous y reviendrons. De même qu'il n'y a dans la nature que certains isomères pour les acides gras, isomères de positions (doubles liaisons en 9-IO-I2-I3 - I5-I6), isomères géométriques (acides gras $c i s$ ), il doit y avoir des types de structure pour les constituants qui tiennent à la spécificité des enzymes qui président à leur synthèse.

\section{DEUXIËME PAR'TIE}

\section{YALEUR NUTRITIVE DES LIPIDES}

Le rôle calorique des lipides n'est plus à discuter ; il est reconnu depuis longtemps ; c'est le constituant de la ration qui a la plus haute valeur calorique : 9,3 calories par gramme à l'état sec contre 4, I pour les protides et les glucides.

Il est également évident qu'indépendamment de leur valeur intrinsèque, les graisses jouent un rôle important dans l'alimentation comme véhicule des vitamines liposolubles, $A, D, E, K$, en même temps qu'elles les protègent contre l'oxydation.

On sait par ailleurs depuis les travaux de BURr et BURR (I929), que certains acides gras désaturés, particulièrement l'acide linoléique, doivent être apportés dans le régime du jeune rat, faute de quoi on observe un retard de croissance accompagné d'une série de troubles en particulier des dermatoses et des hémorragies qui peuvent entraîner la mort. Ces 
troubles sont complètement guéris par administration d'huiles ou de graisses contenant des acides gras désaturés (linoléique, linolénique et arachidonique), ce qui leur vaut le nom d'acides gras indispensables. L,e problème des A. G. indispensables ayant fait l'objet d'une étude récente de E. I E BRETON (23) nous ne l'aborderons pas.

Nous rapellerons seulement que LE BRETON (22) avait émis l'hypothèse que les acides gras indispensables devaient servir à la construction d'un constituant cellulaire (du tissu nerveux) pour expliquer le caractère impérieux qu'ils ont chez l'animal en voie de croissance et que les expériences faites ultérieurement par BEAUVALIET, Brancher, MAY ( 2 bis) sont de nature à étayer cette hypothèse.

En dehors du rôle que les lipides jouent dans la croissance, l'état gravide et la lactation, rôle en rapport surtout avec la teneur en A. G. indispensables du régime, les lipides en général semblent avoir certains effets métaboliques propres, particulièrement vis-à-vis du catabolisme des protéines.

En effet, contrairement aux résultats des anciennes expériencess de L,UsK, des recherches récentes semblent indiquer que les graisses auraient un effet d'épargne plus marqué que les glucides vis-à-vis des protides. Wil,man et coll (39) montrent que lorsque dans un régime sans protéines on réduit l'apport calorique à $50 \mathrm{p}$. Ioo de sa valeur normale, la perte d'azote urinaire reste faible uniquement si le régime contient une quantité suffisante de graisses (20 p. Ioo environ). D'autre part, SAMUEL et coll (33) montrent que la survie des rats soumis au jêtne est plus longue s'ils reçoivent dans les jours précédant la période de jeûne, un régime riche en graisses.

Nous ne nous étendrons pas davantage ici sur l'aspect métabolique et nous terminerons cet aperçu du rôle des lipides dans la nutrition en envisageant rapidement quelques facteurs susceptibles d'influencer l'utilisation des graisses alimentaires par l'organisme.

\section{a) Digestibilité.}

Il est bien évident que la valeur nutritive des lipides est fonction de leur digestibilité. Il semble d'après les résultats des tests de LoNGWorTHY et Hormès (20) et de ceux de DEUEL et coll (I2), que les coefficient de digestibilité : $\frac{\text { quantité de graisse absorbée }}{\text { quantité de graisse ingérée }}$, soit pratiquement identique pour la plupart des graisses animales ou végétales, ainsi que pour certains mélanges industriels testés.

Il est généralement admis que la digestibilité d'une graisse est fonction de son point de fusion (TERrorne (37)) ; si celui-ci dépasse $50^{\circ} \mathrm{C}$, la graisse devient indigeste. Les résultats obtenus par différents auteurs ne 
sont pas toujours concordants, les conditions expérimentales n'étant pas comparables, soit parce que les quantités absolues des graisses ingérées diffèrent, soit parce qu'on ne tient pas compte des quantités de savons excrétées, dans les fèces. Cependant comme le soulignent HoAGLAND et SNIDER (I5) il n'existe pas de relation définie entre le point de fusion d'une graisse et sa digestibilité ; la graisse de mouton qui fond à $47^{\circ}$ par exemple est beaucoup mieux digérée que le beurre de cacao dont le point de fusion n'est pourtant que de $28^{\circ}$.

Il est donc nécessaire de tenir compte en outre de la composition de la graisse. Il est vraisemblable que, non seulement la nature des A. G. entrant dans la constitution d'une graisse intervient dans son utilisation, mais aussi la manière dont les $\mathrm{A}$. G. sont disposés dans la molécule de triglycéride. Les expériences de MATTIL et Higgins (27) sont particulièrement démonstratives à cet égard.

Enfin et le fait est très général, la digestion et l'absorption d'une graisse est fonction non seulement des autres lipides quil'accompagnent, mais aussi de la présence des différents constituants de la ration: sels minéraux, protéines, substances émulsionnantes, etc...

\section{b) Ranciditó.}

On sait que le fait d'exposer une huile ou une graisse à l'air, à la chaleur et à la lumière peut donner à celles-ci une odeur ou une saveur désagréables; les graisses ne sont plus appétissantes et peuvent devenir toxiques. On dit communément qu'elles rancissent. En fait, les altérations qui se produisent peuvent être de nature très différente; il peut s'agir d'hydrolyse, d'oxydation ou d'action microbienne. I,e terme de rancidité a donc une signification différente selon les cas. On ne saurait évidemment en aborder l'étude ici (voir l'article de HoLman (I6)). I es auteurs ne sont d'ailleurs pas d'accord sur le processus chimique en cause. Nous dirons seulement un mot sur l'aspect nutritionnel de la question. D'une part au cours des premiers stades d'oxydation des graisses rances il y a destruction de vitamines (A et $\mathrm{D}$ en particulier) (I6). Et lorsqu'on donne des graisses rances dans le régime à l'animal, il semble qu'il y ait aussi destruction de vitamines hydrosolubles, biotine au niveau de l'intestin et vitamine $\mathrm{C}$.

D'autre part, la rancidité semble due principalement à l'oxydation des A. G. désaturés et ils sont d'autant plus oxydables qu'ils sont plus désaturés. Il y a donc destruction des A. G. indispensables au cours du phénomène.

En fait, les expériences récentes de Holman et GrEEnBERg (I6) montrent que les produits d'oxydation de l'acide linoléique sont inaptes à guérir l'acrodynie du rat due à la carence en acides gras indispensables. 
D'autre part, les recherches de BERNHEIM, WILBUR et KEnASTON (3) montrent que l'oxydation des $\mathrm{A}$. G. désaturés cause une diminution d'activité des enzymes mitochondriaux impliqués dans l'oxydation des graisses, succinoxydase, cytochromoxydase et choline oxydase.

\section{c) Incidences hiologiques ou physiologiques du traitement industriel des matiòres grasses alimentaires.}

I a question vient d'être posée par Wol. F dans un article récent (40). L'auteur constate d'abord que certains constituants des huiles naturelles comme les lecithines et les antioxygènes normalement présents ainsi que certaines vitamines, disparaissent ou sont en partie détruites au cours de la neutralisation et de la décoloration des huiles. D'autre part, il se produit des isomérisations au cours de ces traitements qui sont faits à des températures atteignant $25^{\circ}$. Ces isomérisations n'ont pas seulement pour effet de diminuer la teneur en $\mathrm{A}$. $\mathrm{G}$. indispensables des huiles naturelles, mais elles font apparaitre des A. G. anormaux ayant des doubles liaisons conjuguées et des isomères variés, géométriques ou de position en nombre d'autant plus élevé que les acides gras sont plus désaturés à l'origine.

Nous avons dit précédemment que les résultats des études faites sur la valeur nutritive comparée des beurres, margarines et produits hydrogénés étaient souvent contradictoires et ne permettaient donc pas d'établir une distinction à cet égard entre les matières grasses naturelles et les matières grasses industrielles; cependant de très nombreux travaux ont montré que les huiles de sardines notamment qui sont polymérisées à haute température devenaient fort indigestes.

Par ailleurs, au cours de nos propres recherches sur la digestion des graisses (9) nous nous sommes aperçus incidemment que l'attaque des glycérides par le suc pancréatique est nettement moins rapide et moins complète lorsque le substrat est constitué par une huile ou une graisse en provenance du commerce que lorsqu'il s'agit d'une huile extraite par nousmême au laboratoire à partir de la graine. Le fait doit tenir à notre avis, à une modification de structure qui se produit au cours du traitement industriel. On sait en effet que, au cours des opérations de raffinage par exemple, on réincorpore souvent dans l'huile neutralisée des acides gras provenant des pâtes de neutralisation qui sont réestérifiés ensuite à la glycérine en présence de catalyseurs. Ces glycérides de synthèse ne présentent plus alors leur polymorphisme naturel mais deviennent des glycérides homogènes.

Un autre type de modification de structure, concernant les acides gras cette fois, vient d'être rapporté tout récemment par MABRouk et BRown (24). Ces auteurs américains font état de recherches faites au moyen de la spectrophotométrie infra-rouge sur des margarines et shor- 
tenings. Ils trouvent dans ces produits des proportions d'acides gras trans atteignant parfois $40 \mathrm{p}$. Ioo des acides gras totaux.

Toutes ces constatations montrent que les matières grasses qui ont subi certains traitements industriels perdent en partie leur structure d'origine pour donner des composés qui ne se rencontrent presque jamais dans les produits naturels.

Nous ne voulons pas ranimer à cette occasion de vieilles discussions sur le pouvoir cancérigène des graisses chauffées et oxydées puisque d'ailleurs les résultats des expériences de Rofro (32) faites il y a une quinzaine d'années semblent devoir être annulés par ceux des expériences plus récentes de KIRBy (I g).

Par ailleurs, les Américains qui consomment depuis des années des quantités considérables de margarines et de shortenings ne paraissent pas jusqu'à présent avoir présenté des troubles quelconques. L,eur organisme doit seulement contenir une certaine quantité d'acides gras trans à la place d'acides gras cis. Mais il faut reconnaître que ces matières grasses qui sont ainsi livrées à la consommation, surtout en Amérique, sont de véritables graisses de synthèse. Certes, les opérations de raffinage et d'hydrogénation constituent de réels progrès techniques mais il y aurait peutêtre lieu d'en user avec modération, car, si les produits obtenus sont remarquablement stables, leurs caractères s'écartent vraiment beaucoup de ceux des produits naturels et nous dirons seulement pour conclure, que nous nous associons pleinement ici à l'opinion exprimée par WOLFF (40) à savoir que dans " l'industrialisation des produits destinés à l'alimentation humaine il vaut peut-être mieux être timoré qu'imprudent ".

\section{RÉFÉRENCES BIBLIOGRAPHIQUES}

(I) Ahrens (L. S.) and Craig (L. C.). - J. Biol. Chem., I952, 195, 299.

(2) BAILEX (A. E.). - Industrial Oil and Fat Products, and ed., Interscience Pub. Inc., New York, I95I.

(2 bis). - Beauvallet (M.), Blancher (G.) et May (P.). - J. Physiol., I953, 45, 29 .

(3) BernheIM (F.), Wirbur (K. M.) and Kenaston (C. B.) - Arch. Biochem. Biophys., I952, 38, II7.

(3 bis) Boldingh. - Rec. Trav. Chim., r950, 69, 247.

(4) Borgstrom (B.). - Acta Physiol. Scand., I952, 25, IoI et III.

(5) Borgstrom (B.). - Acta Physiol. Scand., I954, 30, 23 I.

(6) Burr (G. O.) and Burr (M. M.). - J. Biol. Chem., I929, 82, 345.

(7) Caminade. - Bull. Soc. Chimie Biol., ig22, 4, 6oI.

(8) Clément (G.), Clément (J.) et Louedec (A.). - Arch. Sci. Physiol., I954, 8, 233 .

(9) Clément (G.) et Clément (J.). - Arch. Sci. Physiol., I956, 10, 73.

(ro) COOLEy KoEHN. - Anal. Chem., r950, 22, 322.

(II) Desnuelize (P.) et NaUdet (M.). B Bull. I. T. E. R. G., I953.

(I2) Deuel (H. J. jr) and Holmes (A. D.). - U. S. Dept. Agric. Bull., I 922 (27th July), no I033. 
(i3) Dubouloz (P.), Monge-Hedde (M. F.) et Fondarai (J.). - Bull. Soc. Chimie Fr., I947, 800, 900.

(I4) Hilditch (T. P.). - The Chemical Constitution of Natural Fats, 2nd ed., Chapman and Hall Ed., London, I949.

(I5) HoAgrand (R.) and SNider (G. G.). - J. Nutrition, I943, 25, 295.

(I6) Holman (R. T.), Lundberg (W. O.) and MaLkin (T.). - Progress in the Chemistry of Fats and other Lipids, vol. II, Pergamon Press. L.T.D., London, 1954.

(I7) Howard (G. A.) and Martin (A. J. P.). - Biochem. J., I950, 46, 532.

(I8) James (A. T.) and Martin (A. J. P.). - Biochem. J., I954, 57, v.

(Ig) Kirby (A. H. M.). - Brit. J. Cancer, I948, 2, 70.

(20) Langworthy (C. F.) and Holmes (A. D.). - U. S. Dept. Agric. Bull., IgI7 (24th March), no 507.

(2I) LEA (C. H.) and RHodes (D. N.). - Biochem. J., I954, 5\%, xxirI.

(22) LE BRETON (E.). - Ann. Nutrition Alimentation, I949, 3, 225.

(23) Lemeland. - Bull. Soc. Chimie Biol., I923, 5, i Io.

(24) Mabrouck (A. F.) and Brown (J. B.). - J. A. O. C. S., I956, 33, 98.

(25) Machebceur (M.) et Dei.sal (J.). - Bull. Soc. chimie Biol., I942, 24, 297.

(26) Machebcevi (M.) et Deisal (J.). - Bull. Soc. Chimie Biol., I943, 25, II 6 .

(27) MatTit, (K. F.) and Higgins (J. W.). - J. Nutrition, I945, 29, 255.

(28) May (P.). - J. Chim. Phys., I952, 49, 464.

(29) Melier. - Oléagineux, I950, 5, 63I.

(30) PAQUot (C.). - J. Rech. C. N. R. S., I947, p. I3I.

(3I) Pien (J.). - Cf. Mises au point de chimie analytique pure et appliquée et d'analyse bromatologique, $2^{\mathbf{e}}$ série, I955, p. 86.

(32) Roffo (A. H.). - Bull. Assoc. fr. Etudes Cancer, I939, 28, 556.

(33) Samuels (L. T.), Gilmore (R. C.) and Reinlcke (R. M.). - J. Nutrition, I948, 36, 639.

(34) Sarazin (J.), Boubbon (G.) et Calver (R.). - Bull. anciens élèves Ecole fr. de Meunerie, I946, $\mathrm{n}^{\circ}$ 94, I20.

(35) Savary (P.) et Desnuelde (P.). - Bull. Soc. Chimie Fr., I954, , 936.

(36) SPERRy (W. M.) and Webi (M.). - J. Biol. Chem., I950, 18\%, 97.

(37) Terroine (E. F.). - Thèse Faculté des Sciences, Paris, I9I9.

(38) Williams (K. A.). - Oils Fats and Fatty Foods, 3rd ed., J. and A. Churchill Ltd, London, I950.

(39) Willman (W.), Brush (M.), Clark (H.) and Swanson (P.). - Fed. Proc., I947, 6, 423.

(40) WOLFF (G.). - - Cf. Mises au point de chimie analytique pure et appliquée et d'analyse bromatologique, I ${ }^{\text {re }}$ série, I953, p. I59.

(4I) WOLFF (G.) et WolfF (J. P.). - Méthodes d'analyse et de contrôle industriel des matières grasses, Dunod éd., Paris, I953. 